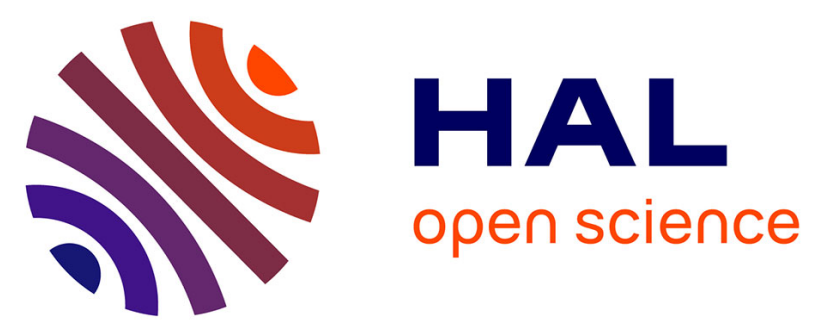

\title{
L'évolution tardi et postglaciaire des lits fluviaux au nord-est de Paris (France). Relations avec les données paléoenvironnementales et l'impact anthropique sur les versants/
}

Jean-François Pastre, Michel Fontugne, Catherine Kuzucuoğlu, Chantal Leroyer, Nicole Limondin-Lozouet, Marc Talon, Nadine Tisnérat

\section{To cite this version:}

Jean-François Pastre, Michel Fontugne, Catherine Kuzucuoğlu, Chantal Leroyer, Nicole LimondinLozouet, et al.. L'évolution tardi et postglaciaire des lits fluviaux au nord-est de Paris (France). Relations avec les données paléoenvironnementales et l'impact anthropique sur les versants/. Géomorphologie: relief, processus, environnement, 1997, 3 (4), pp.291-312. 10.3406/morfo.1997.929 . hal-03357916

\section{HAL Id: hal-03357916 https://hal.science/hal-03357916}

Submitted on 29 Sep 2021

HAL is a multi-disciplinary open access archive for the deposit and dissemination of scientific research documents, whether they are published or not. The documents may come from teaching and research institutions in France or abroad, or from public or private research centers.
L'archive ouverte pluridisciplinaire HAL, est destinée au dépôt et à la diffusion de documents scientifiques de niveau recherche, publiés ou non, émanant des établissements d'enseignement et de recherche français ou étrangers, des laboratoires publics ou privés.

\section{()ㅜ) $\Theta$}

Distributed under a Creative Commons Attribution - NoDerivatives| 4.0 International 


\section{L'évolution tardi et postglaciaire des lits fluviaux au nord-est de} Paris (France). Relations avec les données paléoenvironnementales et l'impact anthropique sur les versants/The evolution of river beds in the North-East region of Paris (France) during the Lateglacial and theHolocene. Relation to the palaeoenvironments and to the human impact on the slopes Jean-François Pastre, Michel Fontugne, Catherine Kuzucuoglu, Chantal Leroyer, Nicole Limondin-Lozouet, Marc Talon, Nadine Tisnérat-Laborde

\section{Citer ce document / Cite this document :}

Pastre Jean-François, Fontugne Michel, Kuzucuoglu Catherine, Leroyer Chantal, Limondin-Lozouet Nicole, Talon Marc, Tisnérat-Laborde Nadine. L'évolution tardi et postglaciaire des lits fluviaux au nord-est de Paris (France). Relations avec les données paléoenvironnementales et l'impact anthropique sur les versants/The evolution of river beds in the North-East region of Paris (France) during the Lateglacial and theHolocene. Relation to the palaeoenvironments and to the human impact on the slopes. In: Géomorphologie : relief, processus, environnement, Décembre 1997, vol. 3, n²4. pp. 291-312;

doi : https://doi.org/10.3406/morfo.1997.929

https://www.persee.fr/doc/morfo_1266-5304_1997_num_3_4_929

Fichier pdf généré le 30/04/2018 


\begin{abstract}
Abstract The dynamics of the river beds in the lower sections of the Marne and the Oise rivers are analyzed in relation to the evolution of the slopes during the Lateglacial and the Holocene. The results of the study of the morphosedimentary responses are confirmed by the palaeoenvironmental variations recorded by malacological, palynological and archaeological data. The Lateglacial evolution appears to be tightly linked to the climatic changes. Two main periods of sedimentation can be attributed to the Pleniglacial I Lateglacial transitional phase and to the Younger Dryas; they are separated by an erosion and soil formation phase which may have occurred during the BOlling and Aherpa phases. At the beginning of the Holocene, an important phase of vertical cutting can be allocated to the first half of the Preboreal period; thereafter river beds tend to be more stable. During the Boreal and the Atlantic phases, the stabilization of the slopes and of the river beds by the vegetation cover plays an important role in the morphogenic evolution. The detrital input remains very low while the river beds are slowly filled in by highly organic material, often dominated by peat formation processes. During the second half of the Atlantic, the various indicators do not show any environmental degradation, but the pollen data show, during the Middle Neolithic times, the expansion of agricultural activities with punctual opening of the landscape and rude- ration. The first episodes of silt sedimentation occur during the second half of the Subboreal, in relation to the erosion of the loess covering the slopes. The river discharges increase during the Bronze Age, thus frequently creating channels which will be filled in during the La Tène period. During the Roman times, the accumulation of sediment in the mean water channels continues in the main valleys; afterwards, the sedimentary dynamics seem to slow down with the decreasing agricultural pressure during the Late Middle Ages. An important erosion crisis occurs during the modern times, marked by an important deposition of silt in the valleys of minor tributaries.
\end{abstract}

\title{
Résumé
}

Résumé La dynamique des lits fluviaux des bassins aval de la Marne et de l'Oise est analysée en terme de réponse à l'évolution des versants durant le Tardi - et le Postglaciaire. Les réponses morphosédimentaires observées sont confrontées aux variations paléoenvironnementales révélées par les données archéologiques, palynologiques et malacologiques. L'évolution tardiglaciaire apparaît étroitement contrôlée par les fluctuations climatiques. Elle comporte deux principales phases de sédimentation, attribuables à la transition Pléniglaciaire-Tardiglaciaire et au Dryas récent et séparées par une phase de creusement et de pédogenèse durant le Bolling et l'AllerOd. L'Holocène débute par une phase de creusement importante au cours de la première moitié du Préboréal, puis les lits tendent à se stabiliser. Durant le Boréal et l'Atlantique, la fixation des versants et des lits fluviaux par la couverture végétale joue un rôle déterminant. Les apports détritiques sont très réduits et les lits subissent un colmatage progressif à forte tendance organique, où les processus de tourbogenèse jouent un rôle important. Durant la seconde partie de l'Atlantique, les différents indicateurs ne semblent pas témoigner d'une atteinte importante du milieu naturel mais les données palynologiques révèlent le développement des activités agricoles du Néolithique moyen traduites par un développement des rudérales et des céréales et une ouverture ponctuelle des paysages. Les premiers colmatages limoneux liés à l'érosion de la couverture loessique des versants surviennent pendant la seconde moitié du Subboréal; puis les écoulements s'amplifient durant l'Âge du Bronze. Ils déterminent l'apparition fréquente de chenaux, qui seront surtout remblayés lors de La Tène. Durant la période romaine, le colmatage des lits mineurs des grandes vallées se parachève, puis les dynamiques sédimentaires semblent se ralentir. Une importante crise d'érosion, marquée par une sédimentation limoneuse importante dans les vallées secondaires est finalement mise en évidence durant la période moderne. 


\title{
L'évolution tardi - et postglaciaire des lits fluviaux au nord-est de Paris (France). Relations avec les données paleoenvironnementales et l'impact anthropique sur les versants
}

\author{
The evolution of river beds in the North-East region of Paris (France) during \\ the Lateglacial and the Holocene. Relation to the palaeoenvironments \\ and to the human impact on the slopes
}

\author{
Jean-François Pastre *, Michel Fontugne ${ }^{\circ}$, Catherine Kuzucuoglu *, \\ Chantal LeROYER ", Nicole LiMONDIN-LOZOUET *, Marc TALON" \\ et Nadine TISNÉRAT ${ }^{\circ}$
}

\begin{abstract}
Résumé
La dynamique des lits fluviaux des bassins aval de la Marne et de l'Oise est analysée en terme de réponse à l'évolution des versants durant le Tardi - et le Postglaciaire. Les réponses morphosédimentaires observées sont confrontées aux variations paléoenvironnementales révélées par les données archéologiques, palynologiques et malacologiques. L'évolution tardiglaciaire apparaît étroitcment contrôlée par les fluctuations climatiques. Elle comporte deux principales phases de sédimentation, attribuables à la transition Pléniglaciaire-Tardiglaciaire et au Dryas récent et séparées par une phase de creusement et de pédogenèse durant le Bølling et l'Allerød. L'Holocène débute par une phase de creusement importante au cours de la première moitié du Préboréal, puis les lits tendent à se stabiliser. Durant le Boréal et l'Atlantique, la fixation des versants et des lits fluviaux par la couverture végétale joue un rôle déterminant. Les apports détritiques sont très réduits et les lits subissent un colmatage progressif à forte tendance organique, où les processus de tourbogenèse jouent un rôle important. Durant la seconde partie de l'Atlantique, les différents indicateurs ne semblent pas témoigner d'une atteinte importante du milieu naturel mais les données palynologiques révèlent le dévcloppement des activités agricoles du Néolithique moyen traduites par un développement des rudérales et des céréales et une ouverture ponctuelle des paysages. Les premiers colmatages limoneux liés à l'érosion de la couverture loessique des versants surviennent pendant la seconde moitié du Subboréal; puis les écoulements s'amplifient durant l'Âge du Bronze. Ils déterminent l'apparition fréquente de chenaux, qui seront surtout remblayés lors de La Tène. Durant la période romaine, le colmatage des lits mineurs des grandes vallées se parachève, puis les dynamiques sédimentaires semblent se ralentir. Une importante crise d'érosion, marquée par une sédimentation limoneuse importante dans les vallées secondaires est finalement mise en évidence durant la période moderne.
\end{abstract}

Mots-clés : Tardiglaciaire, Holocène, versants, lits fluviaux, enregistrements morpho-sédimentaires, occupations humaines, paléoenvironnements, lle-de-France, Picardie.

\section{Abstract}

The dynamics of the river beds in the lower sections of the Marne and the Oise rivers are analyzed in relation to the evolution of the slopes during the Lateglacial and the Holocene. The results of the study of the morphosedimentary responses are confirmed by the palaeoenvironmental variations recorded by

\footnotetext{
* Laboratoire de Géographie Physique, URA 141 CNRS. 1 place Aristide Briand. 92195 Meudon cedex (France)

- Centre des Faibles Radioactivités, avenue de la Terrasse. 91118 Gif-sur-Yvelle cedex (France)

$\checkmark$ Centre National de Préhistoire. UMR 9933 (NRS. 38 avenue du 26" R.I.. 2400) Périgueux (France)

$\triangle$ Association Française pour les Fouilles Archéologiques Nationales. Antenne Nord-Picardic. 516 rue Saint Fuscien $80(190)$ Amiens cedex (France)
} 
malacological, palynological and archaeological data. The Lateglacial cvolution appears to be tightly linked to the climatic changes. Two main periods of sedimentation can be attributed to the Pleniglacial Lateglacial transitional phase and to the Younger Dryas; they are separated by an erosion and soil formation phase which may have occurred during the Bolling and Allerod phases. At the beginning of the Holocene, an important phase of vertical cutting can be allocated to the first half of the Preboreal period; thereafter river beds tend to be more stable. During the Boreal and the Atlantic phases, the stabilization of the slopes and of the river beds by the vegetation cover plays an important role in the morphogenic evolution. The detrital input remains very low while the river beds are slowly filled in by highly organic material, often dominated by peat formation processes. During the second half of the Atlantic, the various indicators do not show any environmental degradation, but the pollen data show, during the Middle Neolithic times, the expansion of agricultural activities with punctual opening of the landscape and ruderation. The first episodes of silt sedimentation occur during the second half of the Subboreal, in relation to the erosion of the loess covering the slopes. The river discharges increase during the Bronze Age, thus frequently creating channels which will be filled in during the La Tene period. During the Roman times, the accumulation of sediment in the mean water channels continues in the main valleys; afterwards, the sedimentary dynamics seem to slow down with the decreasing agricultural pressure during the Late Middle Ages. An important erosion crisis occurs during the modern times, marked by an important deposition of silt in the valleys of minor tributaries.

Key-words : Lateglacial, Holocene, slopes, river beds, morphosedimentary evolution, Human impact, palaeoenvironments, Ile-de-France, Picardic.

\section{INTRODUCTION}

L'évolution tardiglaciaire et postglaciaire du Bassin parisien a été relativement peu étudiée (Belgrand, 1883; Bourdier, 1969). Le développement des recherches liées à l'archéologie de sauvetage au début des années 90 a montré la richesse importante des fonds de vallée (Krier et al., 1991; Pastre et al., 1991). Ceux-ci comportent des enregistrements limono-tourbeux polyphasés d'une épaisseur souvent proche de 10 mètres dont les emboîtements successifs peuvent aboutir à des épaisseurs cumulées de plusieurs dizaines de mètres. L'analyse géomorphologique et chronostratigraphique de ces séquences met en évidence leur relation étroite avec les variations climatiques et anthropiques du milieu. Les données palynologiques et malacologiques apportent une vision couplée des variations paléoenvironnementales et en particulier du couvert végétal. La convergence des résultats qui seront développés ici, montre grosso modo que chaque phase d'apport détritique marque une érosion des interfluves, chaque phase d'incision une modification importante du régime climatique, chaque phase de stabilité fluviatile et d'organogenèse une stabilisation bioclimatique importante du système morphogénétique. Ces « réponses morphosédimentaires ", permettent donc une lecture indirecte des phénomènes érosifs, souvent difficilement caractérisables directement sur les versants.

Le secteur étudié concerne le nord-est de la région parisienne (fig. 1). Les surfaces substructurales faiblement ondulées des plateaux calcaires du Valois (Lutétien, calcaire grossier) et de la Plaine-de-France (Bartonien, marno-calcaire de Saint-Ouen) constituent les unités morphologiques les plus caractéristiques de la région, alors que les formations argileuses ou sableuses yprésiennes et bartoniennes affleurent dans les vallées ou au pourtour des buttes-témoins. Ces unités sont largement recouvertes par des limons loessiques pléistocènes, d'âge essentiellement wcichsélien (Lautridou, 1985), qui constituent une couverture superficielle aisément mobilisable. Le drainage est assuré par des petites rivières qui alimentent deux grands affluents de la Seine : 


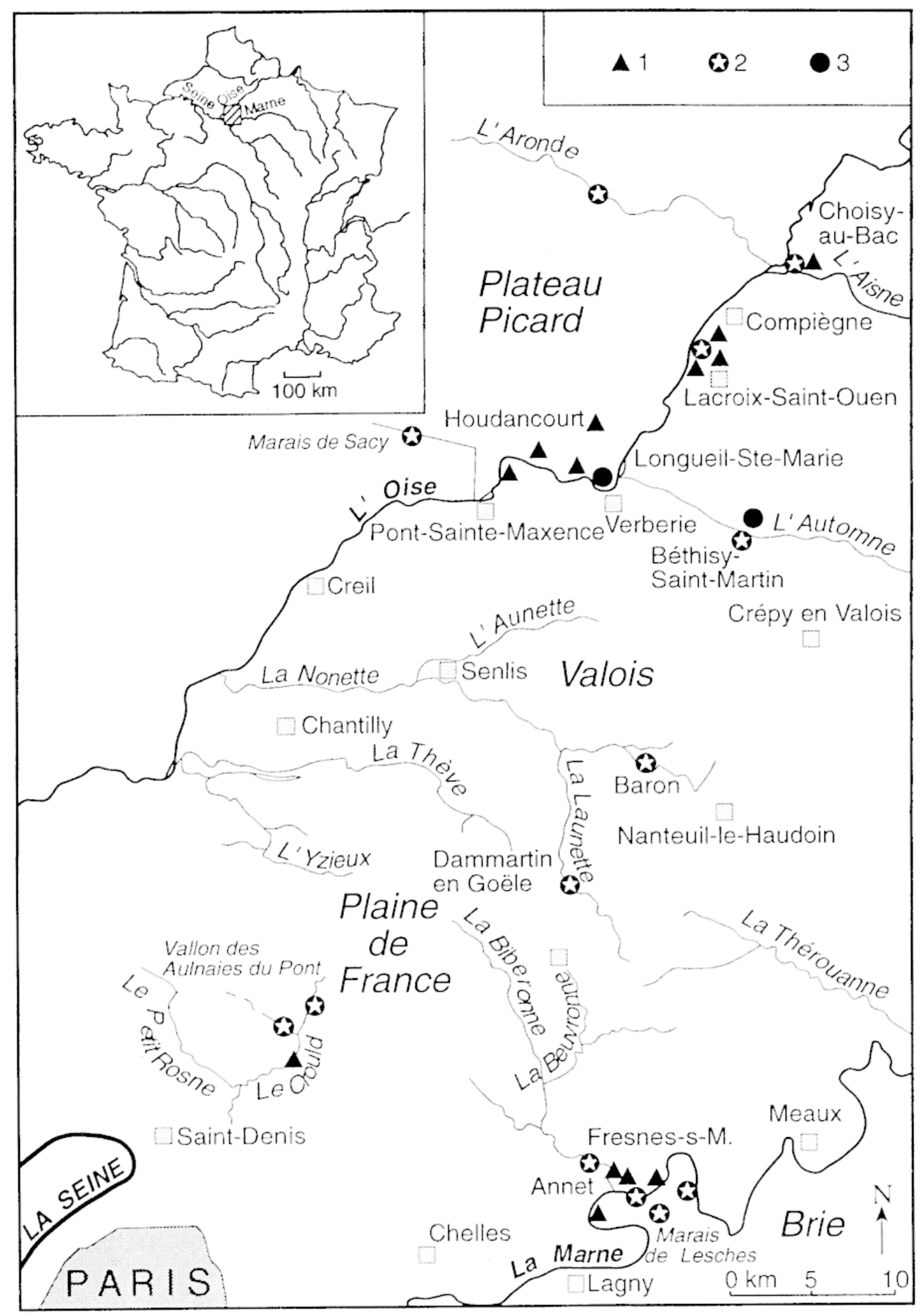

Fig. 1 - Localisation de la région étudiée et des points d'observation. 1 : observations en coupe; 2 : sondages tarière et carottages: 3 : sondages tarière seule
Fig. I-Location of the studied area, outcrops and borings. 1 : outcrops; 2 : corings and highway auger drillings; 3 : single highway auger drillings 
l'Oise et la Marne. Les premières présentent des débits moyens inférieurs à $2 \mathrm{~m}^{3} / \mathrm{s}$, les secondes des débits proches de $90 \mathrm{~m}^{3} / \mathrm{s}$.

Les résultats présentés constituent une première synthèse des données acquises récemment et concernent à la fois les grandes vallées et leurs petits affluents. Ils ont été obtenus dans le cadre des recherches archéologiques réalisées sur le tracé du TGV Nord - Interconnexion, lors de l'exploitation de gravières (vallées de l'Oise et de la Marne) ou à l'occasion de divers travaux d'aménagement. La réalisation de plus de 150 sondages avec une sondeuse "Sédidrill 140 » a permis d'appréhender la géométrie et la stratigraphie d'ensemble des dépôts grâce à des transects perpendiculaires à l'axe des vallées. Ces transects réalisés à la tarière hélicoïdale à un espacement de 10,20 ou 50 mètres ont déterminé l'implantation de carottages destinés aux analyses. De nombreuses tranchées d'évaluation archéologique ou coupes de gravières, ont autorisé une analyse morphostratigraphique plus fine des dépôts, ainsi que l'étude de leurs relations avec les implantations humaines. La présentation des résultats selon un ordre chronologique permet d'analyser en parallèle les relations entre l'évolution des versants, des lits fluviaux et de l'environnement pour chaque période considérée. Le positionnement chronologique des séquences est assuré par des datations radiocarbone sur bois ou tourbe, et par l'utilisation des données archéologiques, palynologiques ou malacologiques. La priorité accordée à l'exposé des données géomorphologiques n'élude pas l'importance des éléments analytiques apportés par ces dernières disciplines et l'exposé de leurs résultats les plus significatifs illustre le caractère pluridisciplinaire de ce type d'approche.

\section{L'IMPACT DYNAMIQUE DES VARIATIONS CLIMATIQUES TARDIGLACIAIRES}

Le Tardiglaciaire constitue un important cycle de modifications des écoulements et des phénomènes d'érosion et de sédimentation. La succession des événements morphosédimentaires enregistrés par les grandes vallées montre leur étroite liaison avec les oscillations climatiques de cette période.

\section{Le début du Tardiglaciaire}

Dans les vallées de l'Oise et de la Marne on observe un important corps sablo-limoneux étendu à l'origine à la totalité des lits majeurs et qui fossilise localement un système de chenaux incisant les cailloutis pléniglaciaires (fig. 2 et 3). Ce dépôt varie en moyenne entre 2 et $3 \mathrm{~m}$ d'épaisseur, ce qui représente un volume moyen de dépôt de $25000 \mathrm{~m}^{3} / \mathrm{ha}$. Il peut atteindre dans le cas de la moyenne vallée de l'Oise une largeur supérieure à $3 \mathrm{~km}$ (fig. 2). Il remanie les sables et les argiles tertiaires et la couverture limoneuse pléistocène, en particulier les loess weichséliens. Ce dépôt traduit une importante phase d'érosion des versants et une sédimentation à caractère saisonnier (probable débacle estivale). L'extension latérale des sédiments, leur organisation (litage, stratification oblique incurvée, figures de chenaux...) caractérisent un réseau en tresse qui évolue localement (spatialement, mais aussi chronologiquement?) vers un système à méandres.

Le début de cette phase de sédimentation reste imprécis par manque d'éléments datables. Si son attribution principale à la période transitoire du Dryas ancien (Oldest Dryas) peut être proposée, on ne peut exclure que certains de ses faciès appartiennent 

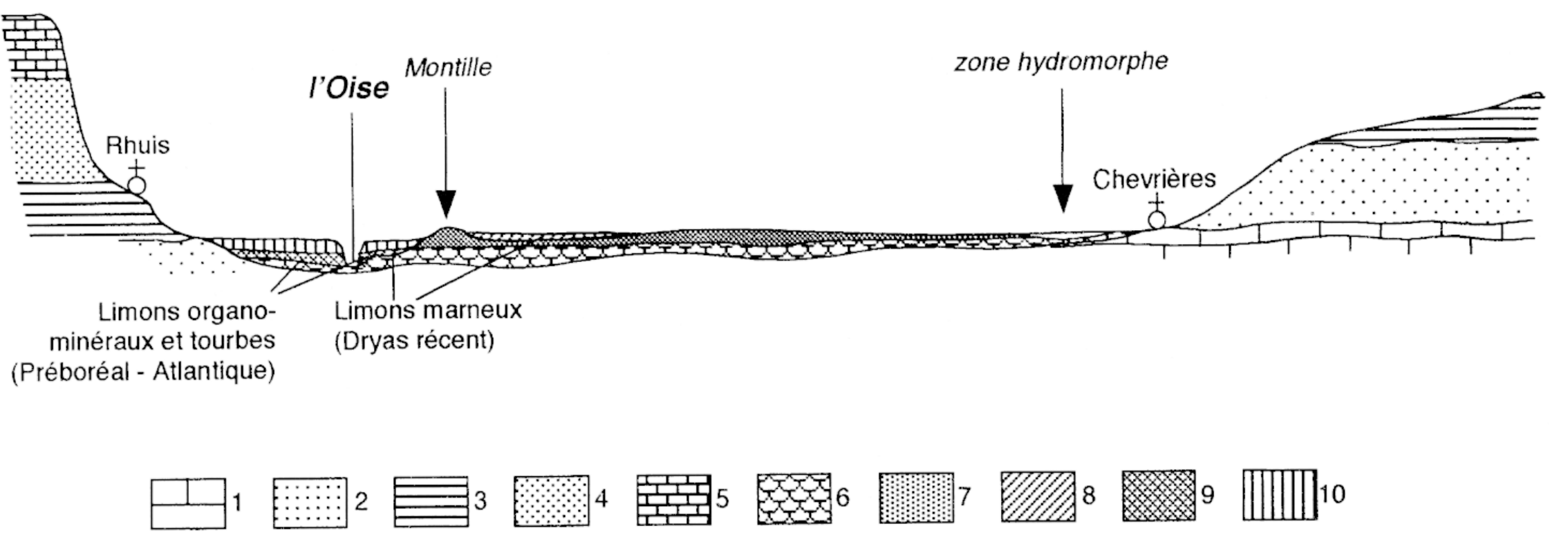

Fig. 2 - Coupe de la vallée de l'Oise aux environs de Verberie (Oise).

1 : craic sénonienne: 2 : sables thanétiens: 3 : argiles yprésiennes (Sparnacien); $4:$ sables yprésiens (Cuisien) : 5 : calcaires lutétiens: 6 : sables et cailloutis weichséliens; 7 : sables et limons (début Tardiglaciaire); 8 : limons marneux (Dryas récent): 9 : limons organominéraux et tourbes (Préboréal-Subboréal): 10 : limons argileux (Subboréal-Subatlantique)

Fig. 2 - Cross section of the Oise valley near Verberie (Oise). 1 : s'nonian chalk: 2 : thanetian sands; 3 : ypresian clays (Lower Ypresian) ; $f$ : ipresian sands (Upper Ypresian); 5 : lutetian limestones; 6 : weichselian sands and gravels: 7 : sands and silts (early Lateglacial) ; 8 : marly silts (Younger Drvas): 9 : organic and mineral silts, peat (PreborealSubboreal): 10 : argillaceous silts (Subboreal-Subatlantic) 

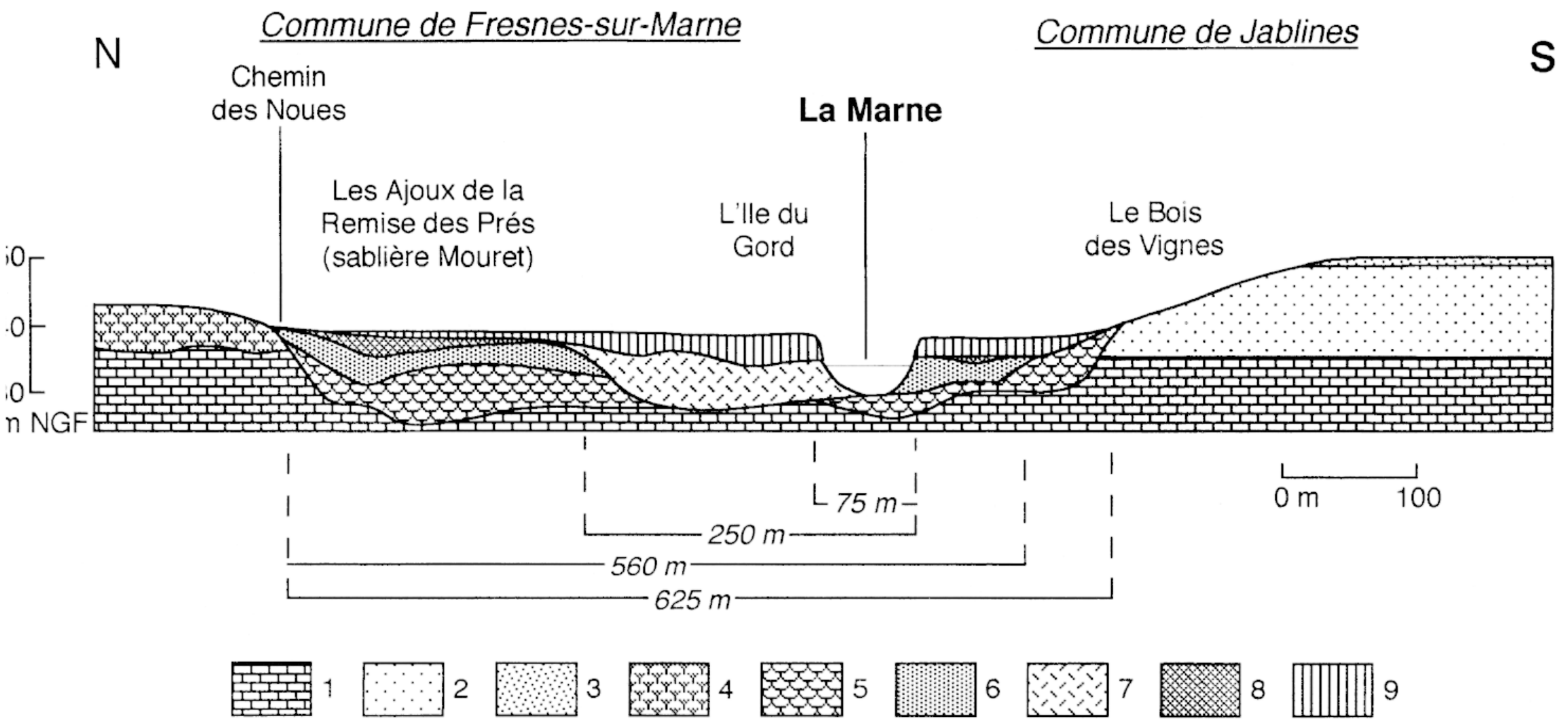

Fig. 3 - Coupe de la vallée de la Marne à l'amont de Fresnes-surMarne (Seine-et-Marne) dans la zone de passage du TGV-Interconnexion. 1 : marnes et calcaires (Lutétien supérieur); 2 : sables de Beauchamp (Bartonien inférieur): 3 : nappe alluviale 1 (cailloutis résiduels) $;$ : nappe alluviale 2 (cailloutis saaliens) $5:$ nappe alluviale 3 (cailloutis weichséliens): $6:$ sables et limons (Dryas ancien): $7:$ tufs calcaires et limons organo-minéraux (Allerod-Atlantique): 8 : tourbes (Preboréal-Subboréal); 9 : limons argileux (Subboréal-Subatlantique)
Fig. 3 - Cross section of the Marne valley upstream Fresnes-surMarne. I: marls and limestomes (Upper Lutetian): $2:$ Beauchamp) sands (Lower Bartonian) : 3 : alluvial terrace 1 (residual gravels) : 4 : alluial terrace 2 (Saalian gravels); 5 : alluvial terrace 3 (Weichselian gravels): 6 : sands and silts (Lateglacial): 7 : travertine and organo-mineral silts

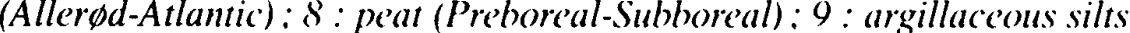
(Subboreal-Subatlantic). 
encore au Pléniglaciaire supérieur, alors que par analogic avec les dépôts associés aux sites magdaléniens de Pincevent et d'Etiolles dans la vallée de la Seine (Roblin-Jouve, 1994) sa terminaison pourrait se situer durant la première moitić du Bølling. Dans la vallée de l'Oise, celle-ci semble ainsi de peu antérieure à l'occupation Paléolithique supérieur de Verberic, datée d'environ 12500 B.P. par ${ }^{14} \mathrm{C}$ (Audouze et al., 1991) et 13000 par thermoluminescence (Valladas, 1981, 1994), Les niveaux magdaléniens reposent en effet sur des sédiments de ce type et sont fossilisés par quelques dizaines de centimètres de sables limoneux affectés par la pédogenèse holocène.

Dans la valléc de la Marne, la malacofaune présente une association d'espèces de phase de transition bioclimatique à Pupilla muscorum, Vallonia et Trichia hispida comparable aux associations reconnues en Angleterre (Kerney, Preece and Turner, 1980); Preece, 1990) ou dans la vallée de la Somme (Limondin, 1995a).

La pauvreté des spectres polliniques et leur pollution par des pollens tertiaires ne permettent pas de disposer de données palynologiques fiables pour cette période.

\section{Le creusement et la stabilisation interstadiaire (Bølling-Allerød)}

La seconde phase marque un ralentissement important des processus sédimentaires et la fin du tressage et des divagations. Les écoulements se concentrent d'abord dans un chenal principal à méandres, éventuellement associé à des chenaux secondaires : 1 à 3 (moyenne vallée de l'Oise). Ces chenaux dissèquent la surface de remblaiement antérieure dont ils isolent ses lambeaux sous forme de bancs sableux et de montilles dont la surface va de quelques centaines de $\mathrm{m}^{2}$ à plus de 50 hectares (fig. 2). Les chenaux secondaires, qui peuvent inciser jusqu'aux cailloutis pléniglaciaires, cessent assez rapidement d'être fonctionnels et la totalité de l'écoulement se concentre dans un chenal unique où le creusement se poursuit.

Ce premier épisode d'incision important semble pouvoir être situé à la fin de la première moitié du Bølling. A La Croix-Saint-Ouen (Oise), des fragments de bois interstratifiés dans un petit banc sableux résiduel localisé dans la zone d'incision, ont donné un âge de $12400 \pm 120$ B.P. (Gif tandétron A. 95545). Les observations similaires effectuées dans les vallées de la Seine (Roblin-Jouve, 1994) et de la Somme (Antoine, 1997 a et b ), de la Petite Nette (Munaut et Paulissen, 1973) et de la Haine (Haesaerts, 1984a et b) en Belgique, de la Meuse aux Pays-Bas (Bohncke et al., 1987; Vandenberghe, 1993, Vandenberghe et al., 1987, 1994) ou de la Warta (Kozarski, 1983; Bohncke et al., 1995) et de la Vistule (Kalicki, 1991) en Pologne, montrent la généralisation de ce phénomène à la majorité des vallées de l'Europe du Nord-Ouest.

Dans la même vallée de l'Oise, à l'aval de Compiègne (La Croix-Saint-Ouen, Longueil-Sainte-Marie, Verberie...), un épisode de sédimentation marqué par le dépôt de limons marneux interrompt un temps ce processus. Caractérise t'il un phénomène de sédimentation à valeur purement locale ou représente t'il l'expression sédimentaire du "Dryas moyen » (Older Dryas)? La question renvoie aux observations similaires effectuées récemment dans le bassin de la Somme (Antoine, 1997 a et b) ou aux variations reconnues dans certains spectres palynologiques de la France du Nord ou de la Belgique (de Ploey, 1963; Munaut et Paulissen, 1973; Woillard, 1975; Emontspohl et Vermeersch, 1991). La malacofaune, pour l'essentiel aquatique, est malheureusement peu représentative des conditions climatiques (fig. 4, LT1). La composition des cortèges (Anisus leucostoma, Armiger crista, Valvata piscinalis, Lymnaea peregra) 
indique un milieu stagnant et très végétalisé. Le taxon terrestre dominant, Oxyloma elegans, est une espèce de milieu très humide (Kerney et al., 1983).

L'incision reprend ensuite brièvement dans l'axe du chenal principal. Elle précède une nette phase de pédogenèse : un gley se met en place sur les limons marneux déposés en berge ou en fond de chenal alors qu'un petit sol humifère se développe sur les zones latérales exondées. Ce paléosol, retrouvé en de nombreux points de la vallée de l'Oise entre Compiègne et Creil ainsi qu'à l'aval (environs de Beaumont-sur-Oise : Krier, communication orale; Pastre, inédit), constitue une entité régionale désormais bien reconnue. Il doit être situé durant l'Allerød, principale phase d'organogenèse de la fin du Pléistocène supérieur dans le nord de la France. Il peut ainsi être parallélisé avec le « Sol de Belloy » identifié dans le bassin de la Somme (Fagnart, 1993; Antoine, 1997 a et b) ou celui découvert récemment à Rueil « le Closeau » dans la vallée de la Seine près de Paris (Bodu et Gebhardt, communication orale - Bodu, 1995 a et b). Ces niveaux étant encadrés par des industries du Paléolithique final de type Federmesser compris entre environ 11600 et 11000 B.P. (Fagnart, 1993; Bodu, 1995). L'importante diminution des apports sédimentaires durant cette période peut être reliée à l'amélioration du régime morphoclimatique, mais aussi à la stabilisation des versants par le développement de la couverture végétale. Ce développement, reconnu sur le plan régional, est marqué par deux phases successives de développement du couvert arboré à pin et bouleau, la dominance du bouleau étant relayée par celle du pin (Van Zeist et Van Der Spoel-Walvius, 1980; Leroyer, 1997)

La faune de mollusques identifiée dans ce paléosol Allerød, des berges de l'Oise à La Croix-Saint-Ouen (station d'épuration), est riche en individus (fig. 4, LT2). Elle est caractérisée par la dominance de trois taxons de zone ouverte (Vallonia pulchella, $V$. costata, Pupilla muscorum) et par la présence de deux espèces de milieu sec, hors de leurs aires de répartition actuelles : Helicopsis striata et Trochoidea geyeri. Les espèces secondaires du cortège sont mésophiles. Cette association indique un milieu ouvert permettant la cohabitation de taxons hygrophiles et xérophiles. Elle est comparable à celles du Closeau dans la vallée de la Seine (Limondin, 1995b) et à celles de la vallée de la Somme (Limondin, 1995a).

Dans les grandes vallées, la tourbogenèse demeure très ponctuelle durant cette période (Pastre, travaux en cours). La formation de tourbe a par contre pu être établie dans certains affluents comme la vallée de l'Automne, petit affluent de l'Oise (fig. 1). A Béthisy-Saint-Martin, une séquence, préservée dans un paléochenal incisé dans les sables du Dryas ancien, montre ainsi de premiers phénomènes de tourbification, correspondant à l'Allerød $(11140 \pm 100$ B.P., Gif -9599$)$, voire peut-être à la base au Bølling. Une évolution proche est observée dans les dépressions comme les marais de Sacy.

\section{Le regain des processus érosifs et sédimentaires au Dryas récent}

Le retour aux conditions froides du Dryas récent entraine un regain important des processus érosifs et sédimentaires.

Dans la moyenne vallée de l'Oise des apports limono-marneux remblayent le chenal principal et les chenaux latéraux sur des épaisseurs variant de 0 à $4 \mathrm{~m}$. Les chenaux secondaires sont ainsi largement fossilisés par des dépôts de décantation périodiques (fig. 2), alors qu'une sédimentation sensiblement plus grossière (limons sableux) 

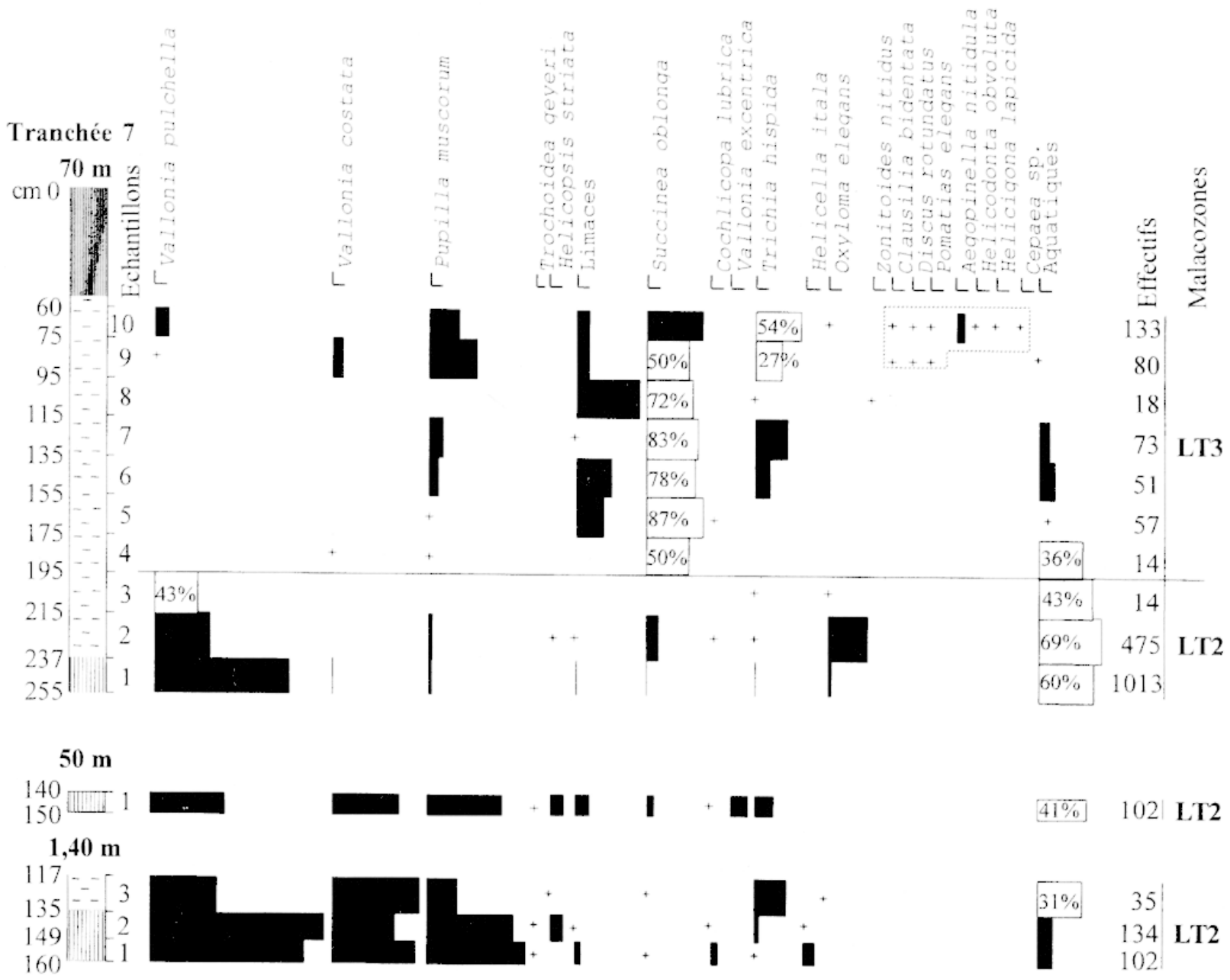

Sondage
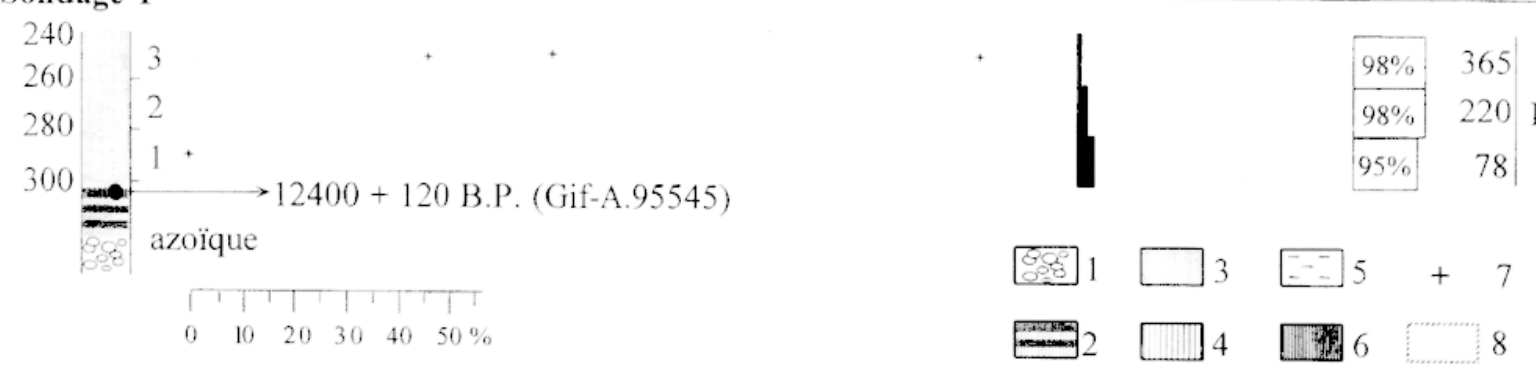

Fig. 4 - Diagrammes malacologiques de la séquence tardiglaciaire de la station d'épuration à La Croix-Saint-Ouen (Oise). 1 : grave weichsélienne; 2 : sable fin lité gris avec lits d'argile; 3 : limon marneux crayeux gris-blanc; 4 : paléosol, silt marneux gris-beige. légèrement humifère; 5 : silt marneux beige à litage diffus; $6:$ sol actuel $; 7:$ un seul individu; 8 : espèces holocènes intrusives.
Fig. 4 - Malacological diagrams from La CroixSaint-Ouen Late Glacial sequence (Oise). 1 : weichselian gravels; 2 : fine grey bedded sand with clay layers; 3 : light grey marly silt; 4 : palaeosoil, raw grey marly silt with low humic contain; 5 : row marly silt with discrete layering; $6:$ actual soil ; 7 : single specimen; 8 : intruded holocene specimens 
caractérise le chenal principal. Le caractère climatique de cette importante réponse sédimentaire est évident. La forte charge crayeuse de ces sédiments accrédite un décapage important des versants amont, la fourniture d'un matériel cryoclastique abondant et l'intervention probable de processus de ruissellement concentré saisonniers relativement développés. La présence discrète de matière organique dans les limons de base témoigne d'une remobilisation probable du sol allerød sur les versants, remobilisation qui s'estompe au plus fort de la sédimentation carbonatée.

La malacofaune est beaucoup moins abondante que dans le paléosol antérieur. Cette faiblesse des effectifs est un indice de la dégradation des conditions environnementales et de la rapidité du comblement. Dans les associations (fig. 4, LT3), Pupilla muscorum se maintient mais les Vallonia, qui sont beaucoup plus thermophiles chutent fortement. Les taxons plus hygrophiles et plus résistants, les limaces, Succinea oblonga et Trichia hispida, forment le groupe dominant, groupe qui constitue la caractéristique principale des malacofaunes du Dryas récent (Limondin, 1995a et sous presse). La fraction aquatique des cortèges devient négligeable. Le paysage est ouvert, les températures se dégradent et seules les espèces à large valence écologique peuvent se développer. L'opposition entre le caractère fluviatile du dépôt et la prédominance des espèces terrestres corrobore la vigueur de la prise en charge sur les interfluves et le caractère saisonnier des apports.

Dans la vallée de la Marne entre Meaux et Lagny, l'enregistrement paraît moins complet. La phase de pédogenèse de l'Allerød n'a pas encore été mise en évidence et l'essentiel de l'évolution sédimentaire semble avoir été confinée dans le chenal principal dès le réchauffement interstadiaire. L'enregistrement y est plus lacunaire et probablement tronqué. Il fait suite à un creusement de plus de 7 mètres, et concerne des limons et des tufs calcaires présentant une association pollinique à Pinus et Betula attribuable à l'Allerød (Leroyer et al., 1997), alors que l'épisode sédimentaire du Dryas récent n'est pas caractérisé.

\section{L'ÉVOLUTION « CLIMATO-ANTHROPIQUE » HOLOCĖNE}

\section{Le creusement du début de l'Holocène}

La phase de creusement holocène, située à la césure Tardiglaciaire-Préboréal, apparaît très rapide, probablement de quelques centaines d'années tout au plus. Elle évacue une partie du colmatage du Dryas récent et incise localement les graves weichséliennes sur plusieurs mètres, atteignant assez fréquemment le substratum tertiaire. Si l'importance de cette incision continue à en faire la phase de creusement principale, il faut toutefois nuancer son ampleur. L'essentiel des observations converge pour montrer que le creusement interstadiaire du Tardiglaciaire a été largement préparatoire et que ce sont la plupart du temps les mêmes lits qui ont été retouchés et réapprofondis. Dans la vallée de la Marne, les cortèges polliniques isolés à la base de la séquence de Fresnessur-Marne (TGV) tendent même à montrer qu'un niveau de creusement similaire était localement atteint au milieu du Tardiglaciaire (Leroyer et al., 1997). Dans les grandes vallées, cette phase de creusement détermine un lit mineur à méandres d'une largeur supérieure au double du lit actuel (fig. 2 et 3). Dans les vallées secondaires, on dénote en général l'individualisation d'un chenal, incisé de 1 à $2 \mathrm{~m}$ dans les sédiments tardiglaciaires. Ce cas est bien illustré par la vallée de la Nonette. La reconstitution qui en 
est proposée (fig. 5) permet d'identifier un chenal bien marqué, colmaté par des limons organo-minéraux contenant de nombreux cônes de Pinus de la seconde moitié du Préboréal $(9250 \pm 100$ B.P. - Gif 9322).

\section{La stabilisation bioclimatique de l'évolution géomorphologique durant la premiè- re moitié de l'Holocène : Préboréal supérieur, Boréal, Atlantique}

Durant la première moitié de l'Holocène, l'extension de la couverture végétale va considérablement ralentir les processus érosifs et sédimentaires et la dynamique fluviale.

Dans les grandes vallées, les apports détritiques se réduisent considérablement dans la seconde moitié du Préboréal et font place au Boréal à une sédimentation organique relativement lente. L'évolution du lit de la Marne durant cette tranche de temps a pu être abordée à Fresnes-sur-Marne par la réalisation d'une série de sondages dans l'ancien lit mineur colmaté et différentes observations dans des chenaux tourbeux latéraux exhumés par des gravières entre Meaux et Lagny (fig. 1). Dans le lit mineur, on note l'apparition de phénomènes organogènes importants relativement précoces. Des limons tourbeux conservés localement donnent des âges ${ }^{1+} \mathrm{C}$ de $9640 \pm 110$ et $9690 \pm 90$ B.P. (Gif 9316 et 9317) et livrent des cortèges polliniques préboréaux à Pinus, Betula et Salix, évoluant ensuite vers des cortèges à Pinus et Corylus (Leroyer, 1995; Leroyer, 1997; Leroyer et al., 1997). L'évolution se poursuit ultérieurement par la mise en place de tufs calcaires et de limons tourbeux présentant des cortèges polliniques à Corylus puis Tilia, caractéristiques du Boréal et de l'Atlantique. Dans les chenaux latéraux hérités du Tardiglaciaire, une tourbification très progressive débute au Boréal.

Dans la moyenne vallée de l'Oise, l'évolution apparaît assez proche. L'aggradation organique qui débute au Préboréal avec des argiles organiques noirâtres, se poursuit jusqu'au Subboréal avec le dépôt de tourbes ligneuses et de limons organiques qui dépassent $2 \mathrm{~m}$ d'épaisseur dans les séquences les plus dilatées. Dans les chenaux latéraux, comparables à ceux de la Marne, on peut noter aussi un développement des tourbes; mais les séquences, d'âge variable selon les secteurs, couvrent des périodes généralement plus limitées.

Ces éléments démontrent la stabilisation importante des lits durant cette période. On est dans une situation "biostasique " type, où le rôle fixateur et régularisateur joué par la végétation apparaît fondamental. La modicité des apports terrigènes traduit la faiblesse de la contribution des versants. L'importance de la productivité organique et la richesse des sédiments tourbeux en débris ligneux démontrent l'existence d'une ripiflore développée qui joue un rôle important dans la stabilité des lits.

Dans les vallées secondaires, l'évolution tourbeuse est quasi générale. On assiste au développement d'importantes tourbières alcalines de bas-marais qui occupent la quasi totalité des fonds de vallées. L'évolution débute en général par des limons organiques qui présentent un cortège floristique typique du Préboréal (Gauthier, 1995; Leroyer, 1995, 1997; Leroyer et al., 1997) et donnent tous des dates ${ }^{14} \mathrm{C}$ comprises entre 9500 et 9000 B.P.; puis des tourbes à Hypnum et herbacées hygrophytes se développent durant le Boréal et l'Atlantique.

Dans les vallées septentrionales tributaires de l'Oise, la tourbification est très progressive. Elle dépasse 3 mètres de tourbe compressée dans les vallées de l'Automne et de l'Aronde. La présence locale de malacofaunes à limnées et planorbes indique des périodes d'inondation, mais il apparaît souvent difficile de discerner dans les enregistrements 

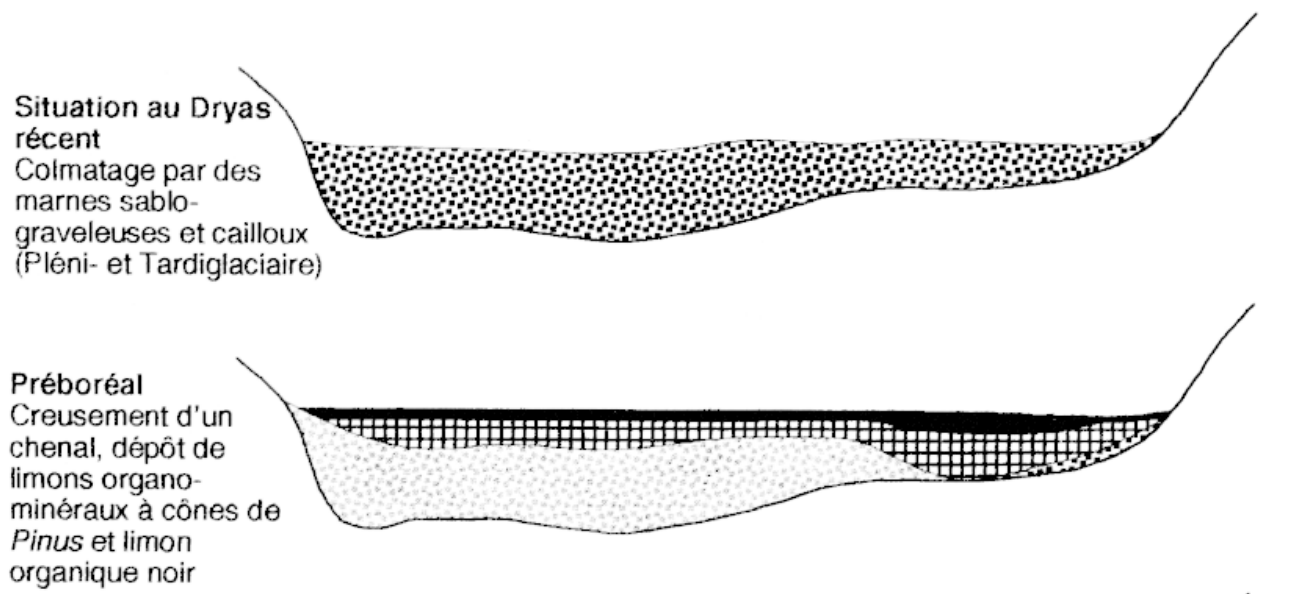

organique noir

Atlantique

Premier épisode

de tourbification
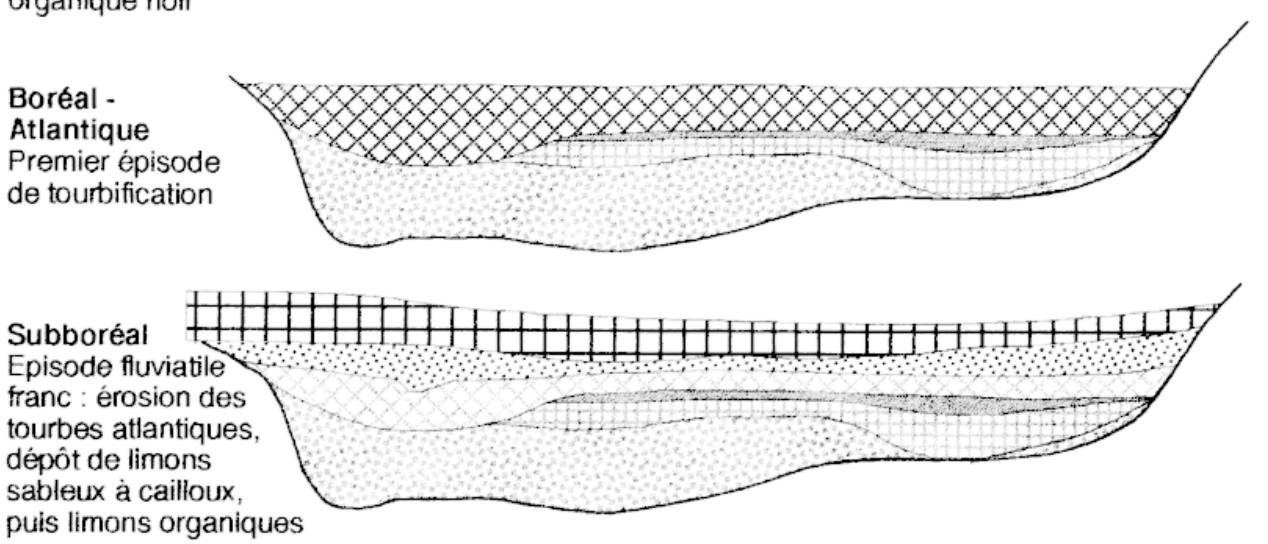

puis limons organiques
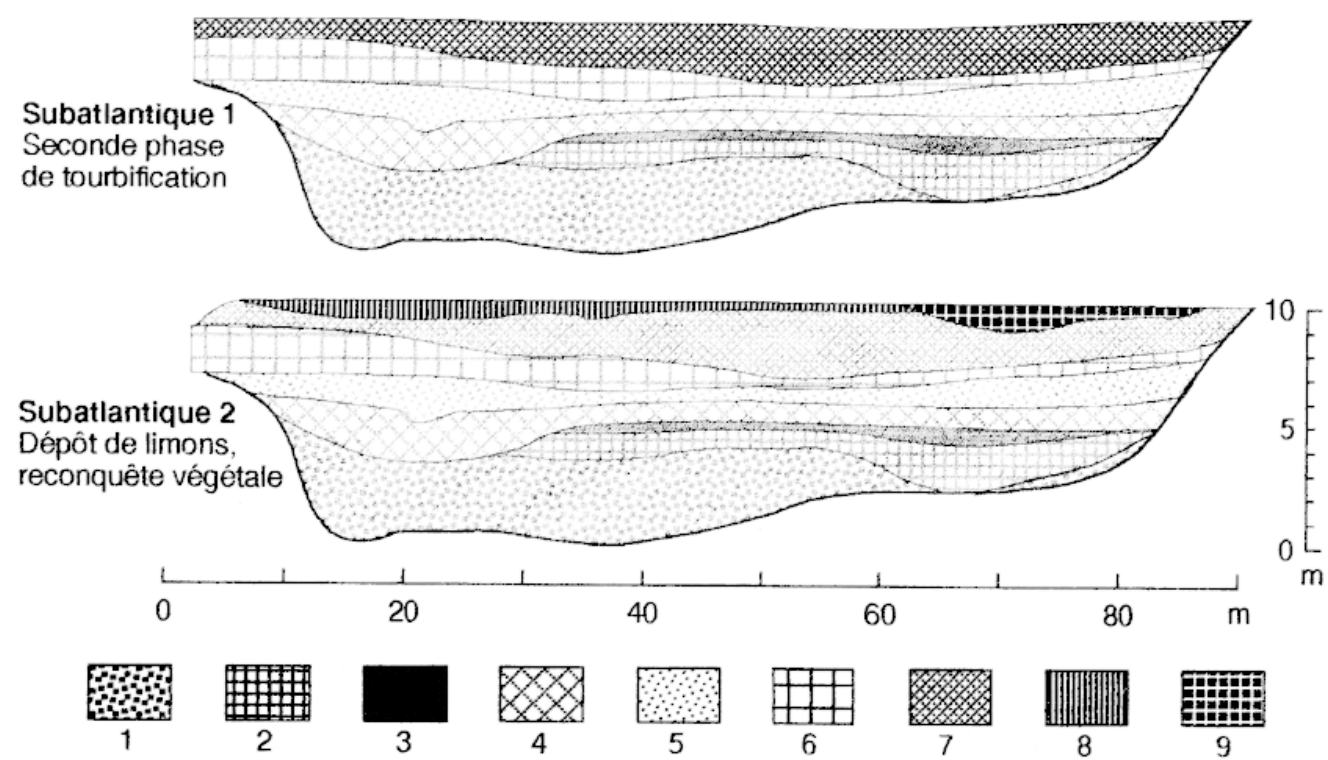

Fig. 5 - Evolution tardi- et postglaciaire de la vallée de la Nonette dans la zone de passage du TGV à Baron (Oise). 1 : sables et limons avec graviers et cailloux calcaires (Pléistocène supéricur); 2 : limons organo-minéraux; 3 : limon humifère noir; 4 : tourbe; $5:$ limons et sables avec cailloux calcaires; 6 : limon organique ; $7:$ tourbe ; $8:$ limon argileux $; 9$ : limon tourbeux

Fig. 5- Late and Postglacial evolution of the Nonette valley along highspeed "TGV-Nord" railway near Baron (Oise). 1 : sands and silts with limestones gravels and pebbles; 2 : organic and mineral silts ; 3 : humic black silt ; 4 : peat ; 5 : silts and sands with limestones; 6 : organic silt; 7 : tourbe; 8 : argillaceous silt; 9 : peaty silt 
morphosédimentaires des chenaux pérennes étendus. L'hypothèse d'un écoulement à dominante hypodermique au sein des tourbes, relayé par des écoulements concentrés superficiels en période de hautes eaux semble la plus plausible.

Dans les vallées de la Plaine-de-France, l'intercalation de tufs calcaires marque des phénomènes liés à des émergences carbonatées locales comme dans la vallée du Crould, ou à des petites précipitations saisonnières comme dans la vallée de la Beuvronne. Le développement des tourbes, surtout actif à l'Atlantique, est moins accentué et les paléoformes indiquent l'existence d'un chenal pérenne bien marqué, bordé par la tourbière.

\section{Les modifications de la fin de l'Atlantique et du début du Subboréal}

La fin de l'Atlantique, ne semble pas porter l'empreinte d'une dégradation irréversible du milieu mais l'impact des activités agricoles du Néolithique moyen y est bien caractérisable. Les spectres polliniques démontrent une ouverture ponctuelle du paysage. Elle est bien caractérisée dans le bassin de la Marne (Leroyer, 1995; Leroyer, 1997; Leroyer et al., 1997) où s'observe une nette poussée des rudérales (fig. 6). Toutefois, l'effet des défrichements du Néolithique moyen ne perturbe pas le bilan érosif au point d'entraîner une modification importante du transit sédimentaire et de ses implications hydrodynamiques. Le régime est pourtant en train de changer. Les sondages réalisés dans le lit mineur de la Marne et les recherches en cours dans celui de l'Oise mettent en évidence une modification de la charge alluviale qui remonte à la fin de l'Atlantique et au début du Subboréal. Les tourbes et les limons organiques font place à des limons organo-minéraux où la charge minérale augmente alors que des intercalations sableuses apparaissent localement. Il y a là la preuve d'une augmentation des apports terrigènes, liée probablement aux effets érosifs des défrichements néolithiques. Cette sédimentation accentue l'exhaussement et l'empâtement des lits mineurs et a pour corollaire un regain de fonctionnement des chenaux latéraux. Dans la vallée de la Marne, à Annet, un bois, contenu dans des sables érodant la tourbe d'un chenal latéral proche du lit mineur, a donné ainsi un âge de $4740 \pm 70$ B.P. (Gif 8457). Les tourbes les plus tardives déposées dans les chenaux livrent des cortèges polliniques subboréaux enrichis en aulne, marquant la partie moyenne du Subboréal $(-3000-4000$ B.P. - Leroyer, 1995; Leroyer, 1997; Leroyer et al., 1997) et dont l'âge est corroboré par une datation ${ }^{14} \mathrm{C}$ de $3740 \pm 90$ B.P. (Gif 8808).

\section{Le développement majeur des processus érosifs et sédimentaires durant la seconde moitié du Subboréal}

Le régime va changer de manière profonde dans la seconde moitié du Subboréal.

Dans la moyenne vallée de l'Oise, la découverte récente de plusicurs sites du Néolithique récent et final au sein des derniers sédiments limono-tourbeux offre des jalons importants. L'afflux de premiers limons argileux libérés par la couverture loessique des versants survient peu après. Dans cette reconstitution, la séquence de la «Station d'épuration » à La Croix-Saint-Ouen près de Compiègne (Talon et al., 1995) fournit de précicux repères. Elle présente des limons tourbeux et des tourbes contenant des vestiges du Néolithique récent (Groupe "Seine-Oise-Marne »), qui sont recouverts par des limons argileux qui commencent à fossiliser la berge de l'Oise (fig. 7). La première unité de ces limons comporte des niveaux d'occupation du Néolithique final de 


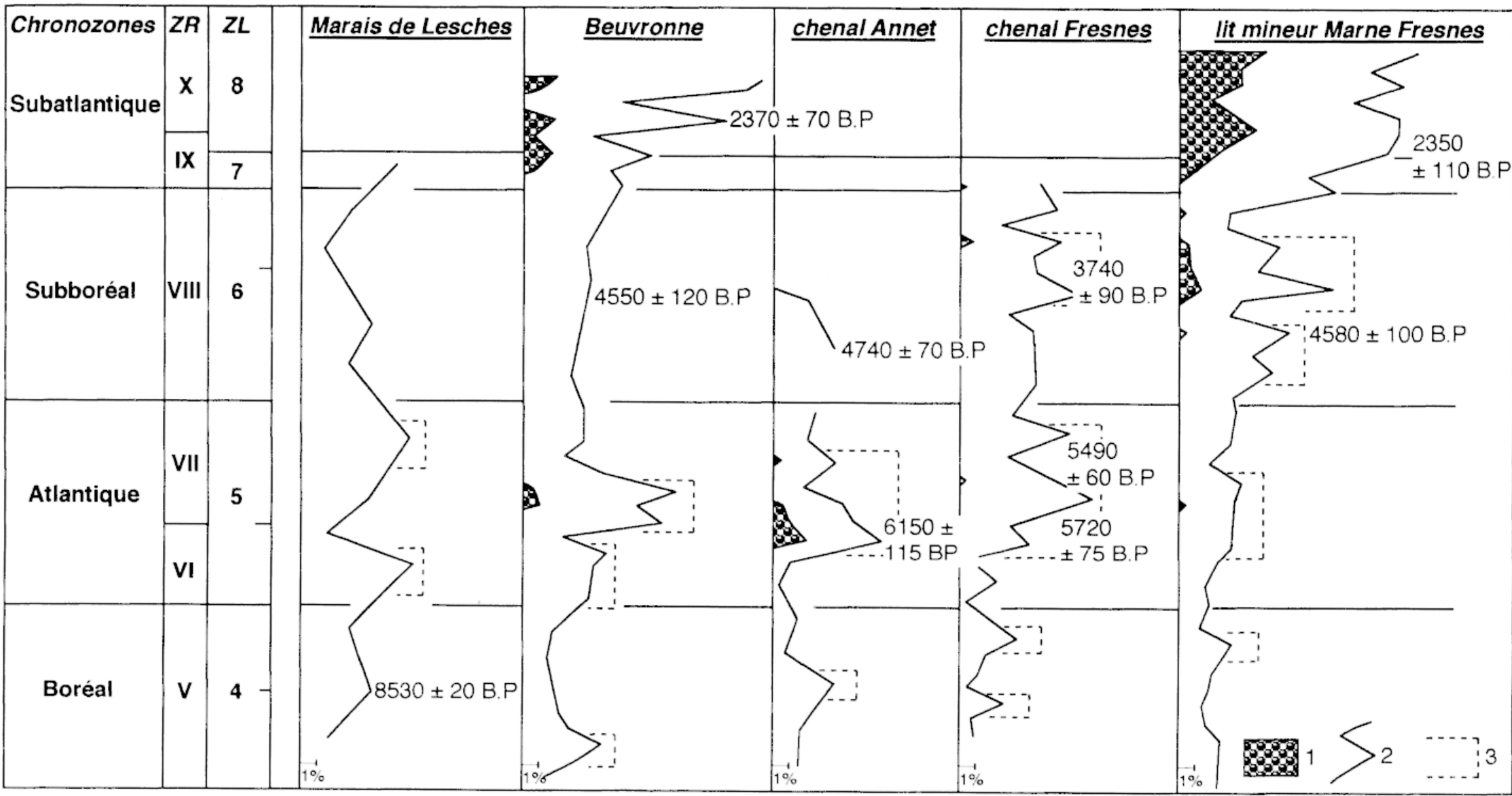

Fig. 6 - Diagramme synthétique d'anthropisation des spectres polliniques de la vallée de la Marne (secteur Meaux-Lagny). d’après Leroyer ét al. (1997). 1 : céréales: 2 : rudérales - 3. phases d'anthropisation : ZR : zones polliniques régionales; ZL : zones polliniques locales.
Fig. o - Synthetic anthropisation diagram according to pollen spectras from the Marne valley between Meaux and Lagny (after Lerover et al.. 1997). I: cereals; 2 : ruderary plants; 3 : anthropisation phases; ZR : regional pollinic zones: ZL. : local pollinic zone's. 

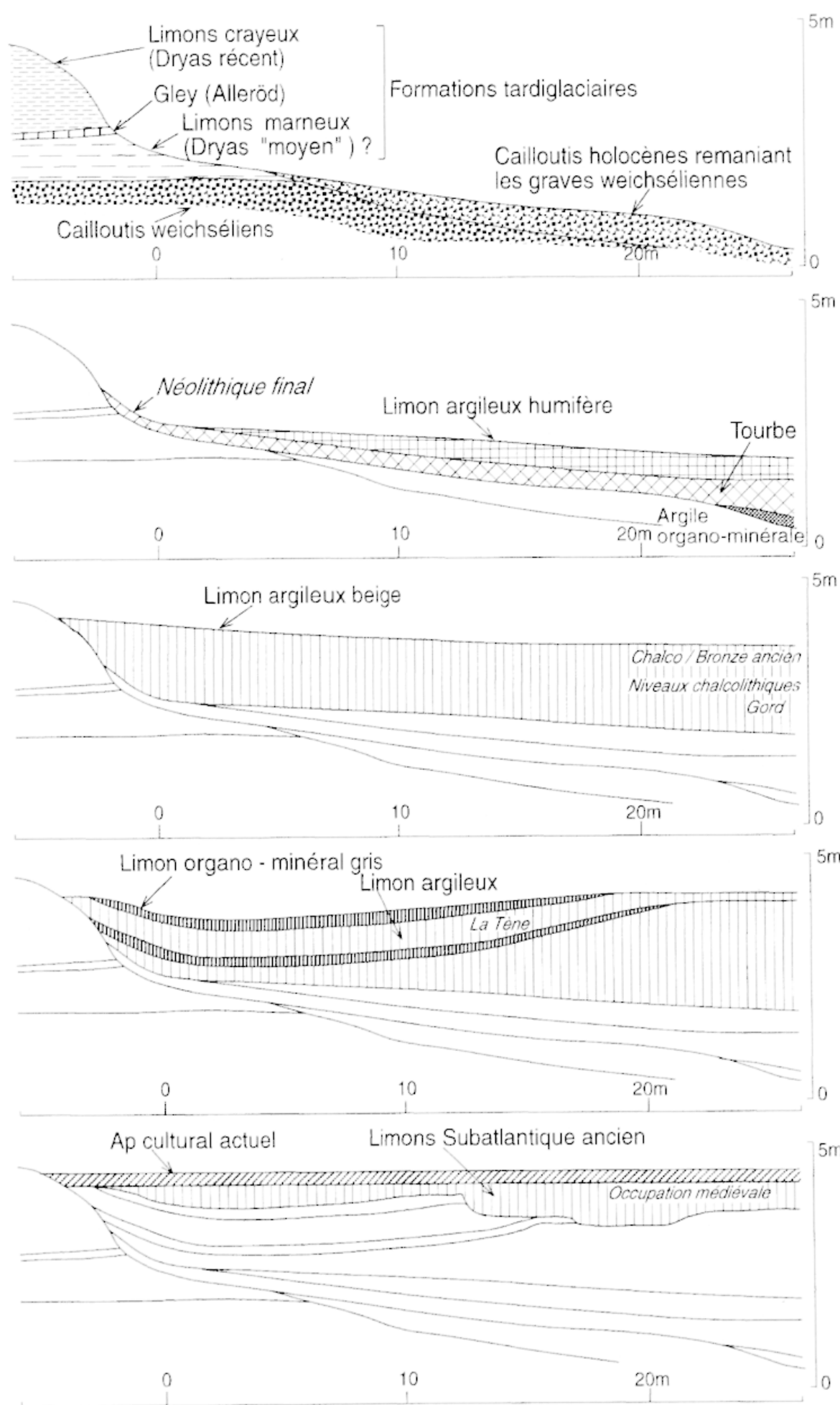

\section{1) Préboréal}

Situation après le creusement holocene Fin de la 1ère moitié du Préboréal. Vers 9500 B.P.

m 2) Fin de l'Atlantique Situation apres le dépôt de sédiments organiques (Préboréal 2, Boréal, Atlantique) Rejets Néolithique final (S.O.M.) $5100-4700$ B.P.

$5 \mathrm{~m} \quad 3)$ Première moitié du Subboréal Dépôt des 1ers limons de "débordement", Occupations chalco Gord et Bronze ancien. 4700 - 4400 B.P. $4000-3800$ B.P.

$5 \mathrm{~m}$ 4) Seconde moitié du Subboréal Situation après le creusement du chenal latéral. Occupation de la Tene ancienne. 2500 B.P.

$5 m$ 5) Subatlantique actuel Colmatage terminal par des limons du Subatlantique ancien. Occupation médiévale, (V-IXème siècle) Culture moderne.
Fig. 7 - Evolution holocène de la berge de l'Oise en rive gauche à $\mathrm{L}$ a Croix-Saint-Ouen (chantier de la station d'épuration, Oise).
Fig. 7 -Holocene evolution of the left bank of the Oise at La Croix-Saint-Ouen (purification station, Oise) 
type Gord et des niveaux du Bronze ancien, qui constituent les jalons chronologiques les plus anciens $(4700-3800$ B.P. env.) reconnus jusqu'alors pour cet ensemble sédimentaire.

La malacofaune des premiers limons déposés dans le lit mineur comporte essentiellement des espèces aquatiques de milieu calme et riche en végétation (Anisus leucostoma, Lymnaea truncatula - fig. 8, LH1a). Ces taxons corroborent la nature fluviatile des apports, mais leur capacité de résistance à l'assèchement ou leur possibilité de remaniement post mortem sont aussi compatibles avec la présence de niveaux d'occupation Néolithique final / Bronze ancien qui indiquent des périodes d'exondation saisonnière. En rive, les associations qui comportent des espèces forestières et hygrophiles témoignent d'un milieu encore riche en végétation, bordé de franges marécageuses (fig. 8, LH1b).

Les modifications morphosédimentaires des lits fluviaux sont nombreuses durant la seconde partie du Subboréal. Dans la vallée de l'Oise, de nombreux chenaux colmatés durant le second Âge du Fer (La Tène) datent de l'Âge du Bronze. Ils dénotent des divagations fréquentes de la rivière, avec des reprises d'incision locales qui marquent un regain de l'activité fluviale. La période $3800-3000$ B.P. semble en particulier avoir été très active. Ceci est bien illustré par le schéma évolutif du site de La Croix-SaintOuen (fig. 7) où on assiste au creusement d'un petit chenal latéral qui sera comblé durarit La Tène. Dans les vallées secondaires, les écoulements s'amplifient. Ils peuvent se traduire par des épisodes érosifs importants. A Houdancourt, la base d'un chenal, creusé par un ruisseau dans les sables tardiglaciaires de la vallée de l'Oise, a livré des bois datés de $3090 \pm 60$ et $3430 \pm 50$ B.P. (Gif 8459 et 8460 ). Ces bois jalonnent le début d'un comblement fluviatile vigoureux, représenté par des sables à stratifications obliques qui précèdent une tourbe contenant des installations du début de la Tène. Sur la série de coupes évolutives de la vallée de la Nonette (fig. 5), on retrouve un épisode fluviatile franc qui tronque les tourbes atlantiques. Dans certaines vallées mineures (Esches, Launette, Aulnaies-du-Pont...) où l'on constate l'absence d'enregistrements sédimentaires avant l'Atlantique, la question d'un «nettoyage » des formations antérieures ou d'une fonctionnalité tardive est posée.

L'origine de ces importants phénomènes d'érosion et de sédimentation peut être discutée, mais les éléments dont on dispose actuellement ne permettent pas d'en discerner encore les causes précises. S'il semble probable que la mobilisation initiale des limons sur les versants est directement liée à l'érosion des terres défrichées, la vigueur soudaine de la réponse sédimentaire dans les grandes vallées surprend et les différents phénomènes hydrodynamiques observés ensuite permettent de s'interroger sur une liaison anthropique simple. L'hypothèse d'une accentuation de la pluviométrie, liée à la péjoration climatique subboréale, constitue peut-être le lien le plus direct entre ces phénomènes, du moins durant sa seconde partie $(-3500 /-2500$ B.P.). Les épisodes d'incision assez violents, comme ccux qui viennent d'être évoqués, semblent en particulier répondre à ce critère. Cette activité hydrodynamique peut être mise en parallèle avec celle mise en évidence dans les bassins du Rhône (Bravard et al., 1991; Bravard, 1992), de la Seine (Krier et al., 1991) ou de la Meuse (Lefèvre et al., 1993). 


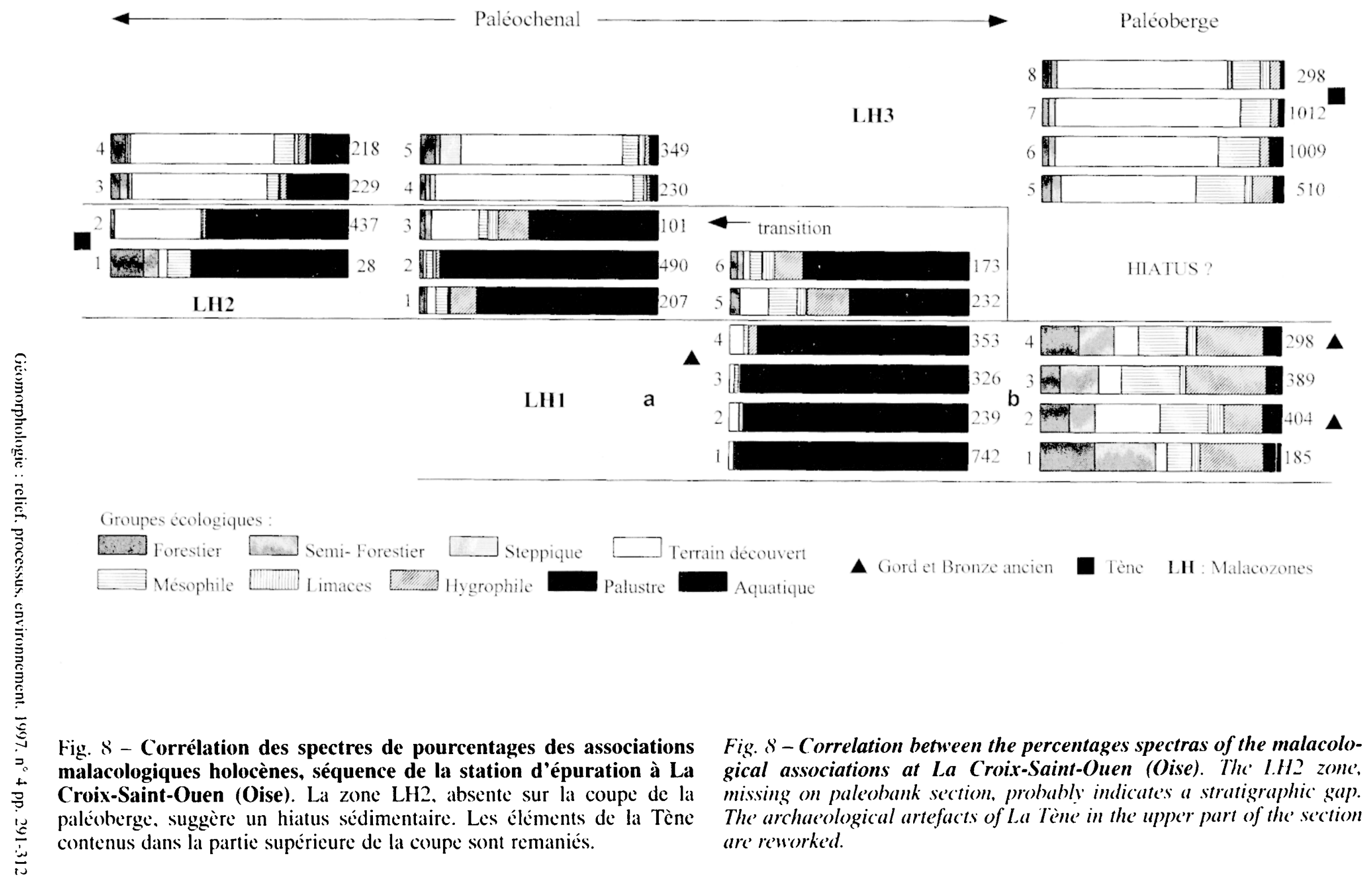

Fig. 8 -Correlation between the percentages spectras of the malacological associations at La Croix-Saint-Ouen (Oise). 'Th' L.H2 zonc', The archaeological artefacts of La Tine in the upper part of the section are reworked. 


\section{Les fluctuations du Subatlantique}

Durant le Subatlantique l'évolution morpho-sédimentaire subboréale se prolonge dans une certaine mesure, mais des modifications assez importantes, qui n'ont été décelées que depuis peu de temps, interviennent aussi.

Dans les vallées de la Marne et de l'Oise, le remblaiement limoneux se poursuit pour donner aux fonds de vallées leur physionomie actuelle et parachever la fossilisation des lits mineurs évasés hérités du début de l'Holocène. Ce processus conduit ainsi à une réduction de la section en travers pouvant atteindre $2 / 3$ des lits mineurs et au développement du système actuel de berges abruptes et instables. Les îles limoneuses se développent. Les chenaux latéraux sont largement remblayés, bien que restant en général légèrement marqués dans la topographie. Cette nouvelle morphologie favorise les débordements en période de crue, et explique en partie la dynamique actuelle.

Les séquences étudiées actuellement sur les grandes vallées permettent de penser que l'évolution a été saccadée. On y observe en particulier des petits paléosols continus et les vestiges archéologiques sont enfouis à des profondeurs très variables en fonction de leur position par rapport au chenal actuel. Dans la vallée de la Marne à Annet, on a découvert des vestiges gallo-romains à proximité du lit mineur actucl, ennoyés sous plus de 3 mètres de limons. Dans la vallée de l'Oise, le site de La CroixSaint-Ouen a permis de découvrir des fours du haut Moyen-âge proches de la berge actuelle, à moins de $50 \mathrm{~cm}$ de profondeur. Ceci montre que la physionomie était alors proche de l'actuelle et que l'aggradation limoneuse s'était poursuivie depuis le second Âge du Fer (La Tène), avec un accès probable durant la période gallo-romaine. L'apparition caractéristique de mollusques de milieu ouvert, dominés par Vallonia pulchella, espèce de zone ouverte assez humide, marque l'importance de la déforestation du fond de vallée (fig. 8, LH3).

Mais c'est surtout dans les vallées secondaires que l'on dispose des jalons les plus nombreux et les plus sûrs. En 1991, nous avions opposé la reprise de l'évolution tourbeuse des rivières de Picardie à « l'ennoiement » limoneux des vallées d'Ile-de-France (Pastre et al., 1991). Une certaine régularité de l'évolution depuis le Subboréal semblait alors se dégager. Or, l'analyse plus précise des phénomènes et l'exploitation de nouvelles séquences révèlent en fait une évolution contrastée.

Le regain des processus tourbeux, dans les vallées comme celles de la Nonette, n'est pas limité aux seules vallées tourbeuses. Certains vallons, qui ne possèdent pas d'enregistrements antérieurs, connaissent des phénomènes de tourbification tardifs. Un des exemples les plus caractéristiques est fourni par le vallon des Aulnaies-du-Pont, à Goussainville près de Roissy (fig. 1), où la tourbification commence seulement au début du Subatlantique ( $2480 \pm 50$ B.P., Gif 9318$)$. Faut-il y voir le seul résultat de l'ouverture du milieu (réduction de l'évapotranspiration, accentuation du transit linéaire...), l'effet de remontées de nappe, le rôle d'aménagements ou plus tardivement la conséquence de la déprise agricole médiévale? Au stade actuel des recherches, la question reste à préciser.

Quant au colmatage limoneux, il apparaît souvent beaucoup plus tardif que celui des grandes vallées. Dans la vallée de l'Esches, petit affluent de l'Oise près de Méru, les alluvions tourbeuses qui contiennent du matériel du haut Moyen-âge sont recouvertes sur l'ensemble du fond de vallée par plus de $2 \mathrm{~m}$ de limons repris à la couverture 
loessique des versants, mais elles contiennent aussi du matériel gallo-romain, ce qui laisse croire à une évolution très limitée durant le Moyen-âge. Dans le vallon des Aulnaies-du-Pont, les derniers sédiments humifères qui précèdent les limons supérieurs ont fourni une datation de 570 \pm 50 B.P. (1304-1434 A.D. cal., Gif - 9319). Dans les autres vallées, on ne dispose pas encore de datations, mais les contextes stratigraphiques suggèrent un signal d'âge similaire. Ces données corroborent ainsi l'existence d'une importante érosion des versants après le Moyen-âge. Elle y est directement caractérisable. L'étude de plusieurs sites archéologiques du Moyen-âge situés en bas de versant (Kuzucuoglu et al., 1992 a et b), témoigne ainsi d'importants colluvionnements après l'occupation de ces sites, avec des tonnages moyens exportés supérieurs à $2 \mathrm{t}$ /ha/an.

Les données chronologiques placent l'essentiel de ces processus colluviaux durant le Petit Âge glaciaire. Si la conjugaison du développement des cultures lié à l'expansion démographique et les effets secondaires de la dégradation climatique fournissent les causes générales de cette évolution, celle-ci reste à analyser plus en détail, tant dans sa chronologie que dans ses processus. Plusieurs vallées, comme la Beuvronne ou la Nonette, montrent par exemple une couche de limon subcontinue qui repose sur les tourbes subatlantiques et marque un accès des apports issus des versants. Cette couche précède localement le développement de sols tourbeux ou humifères avec des horizons A bien développés qui indiquent une diminution des apports après la crise principale et une reconquête par la végétation qui se poursuit actuellement.

La différence qui oppose cette « crise », largement marquée dans les vallées secondaires à la « crise » subboréale, avant tout perçue dans les grandes vallées, peut traduire à la fois une ampleur différente des phénomènes érosifs et un enregistrement contrôlé différemment par l'évolution des lits fluviaux. Pour la première, le « réceptacle » formé par les lits mineurs évasés des grandes vallées a pu absorber, piéger, une partie de la charge évacuée de manière progressive par les rivières secondaires. Pour la seconde, l'engorgement déjà important des lits des grandes vallées n'a pu permettre qu'une aggradation verticale, étendue à la quasi totalité du lit majeur, et entraîner une surcharge sédimentaire des écoulements (de la charge en suspension en particulier). L'importance de la sédimentation récente, mise en évidence dans les petites vallées à environnement limoneux, suggère une déstabilisation beaucoup plus importante des sols, qui peut traduire à la fois une ouverture plus importante des paysages et un impact déterminant des labours.

\section{CONCLUSION}

Ces résultats permettent de définir les grandes phases d'évolution des versants et des lits fluviaux au Tardi - et au Postglaciaire dans la partie nord et nord-est de la région parisienne. S'ils représentent une progression importante par rapport aux données antérieures, ils n'en restent pas moins une étape dans l'élaboration d'un schéma plus précis et mieux interprété.

Les phases d'érosion des versants sont marquées corrélativement par des réponses sédimentaires très nettes dans les vallées, alors que la stabilisation du système lors des phases d'expansion du couvert végétal renforce la valeur biorhexistasique du " modèle » proposé. Si les phases majeures d'évolution sont enregistrées dans la plupart des vallées, et les épisodes spécifiques répercutés par la majorité des émissaires de même 
type, il convient aussi de souligner la variabilité des réponses à moyenne échelle, selon les différents secteurs morphostructuraux d'une même vallée.

Durant le Tardiglaciaire, la « commande climatique » joue un rôle déterminant. Le début du Tardiglaciaire et le Dryas récent sont ainsi marqués par une surcharge sédimentaire importante qui peut être mise en relation directe avec l'ampleur de la mobilisation sur des versants largement dénudés et soumis à la cryoclastie. Le Dryas récent se signale en particulier par une crise notoire qui enraye la stabilité morphologique de l'Allerød.

Pendant la première moitié de l'Holocène, le rôle fixateur joué par la végétation apparaît déterminant. La charge détritique quasiment nulle et la sédimentation organique constatées dans les vallées témoignent de la grande stabilité des versants. Lors de la seconde moitié de l'Holocène, les épisodes érodo-sédimentaires liés aux grandes phases d'expansion agricole et aux fluctuations climatiques sont nettement marqués. Toutefois, les deux causes interfèrent largement et des variations apparaissent dans les réponses en fonction de facteurs locaux. Si le facteur anthropique semble jouer le rôle préparateur de la phase d'érosion de la première partie du Subboréal, l'intervention d'une pluviométrie accentuée constitue peut-être son révélateur. Elle explique en tout cas probablement l'importance du transit fluviatile mis en évidence durant l'Âge du Bronze. Une différence importante distingue le remblaiement limoneux subboréal, qui apparaît relativement progressif dans les grandes vallées, de l'ampleur du colmatage limoneux de nombreuses petites vallées depuis le $X V^{e}$ siècle. La vigueur de l'érosion des terres labourées, l'ouverture du paysage, semblent alors jouer un rôle déterminant exacerbé par la détérioration climatique du Petit Âge glaciaire.

Ces résultats, qui ont le mérite de bénéficier d'un contexte d'enregistrement relativement riche, sont en accord général avec les grandes phases d'évolution constatées dans des contextes géomorphologiques proches en Europe nord-occidentale (voir par exemple Starkel et al., 1987; Frenzel et al., 1995). Ils témoignent cependant aussi de nombreuses variations, liées soit au contexte climato-latitudinal, soit au cadre géomorphologique et lithologique. La recherche d'une plus grande précision devra reposer sur la comparaison entre des séquences plus nombreuses et leur calage chronologique rigoureux. La réalisation d'un bilan morpho-sédimentaire semi-quantitatif, associé à des investigations paléoenvironnementales systématiques, permettra de mieux cerner les causes et l'ampleur des phénomènes.

Nota et remerciements.

Ce texte, prévu pour une publication en 1995 a été complété par une bibliographie actualisée (1997). Les résultats présentés constituent par contre un état des travaux lors du dépôt initial et n'intègrent pas les résultats acquis depuis.

Les auteurs remercient l'Association pour les Fouilles Archéologiques Nationales, la Société Nationale des Chemins de Fer Français et les exploitants de granulats de la moyenne vallée de l'Oise (sociétés Orsagranulats et Redland) pour le concours apporté à leurs travaux.

\section{Références}

Antoine P. (1997a) - Modifications des systèmes fluviatiles à la transition PléniglaciaireTardiglaciaire et à l'Holocène : l'exemple du bassin de la Somme (nord de la France). Géographie physique et Quaternaire, 1997, vol. 51, $\mathrm{n}^{\circ} 1,93-106$.

Antoine P. (1997b) - Evolution Tardiglaciaire et Holocène de la moyenne vallée de la Somme. In Le Tardiglaciaire en Europe du Nord-Ouest, Fagnart J.-P. et Thévenin A. Ed. Editions du CTHS, Paris, 13-26. 
Audouze F., Cahen D., Keeley L.H., et Schmider B. (1991) - Le site magdalénien du Buisson Campin à Verberie (Oise). (jallia Préhistoire, Paris, 24, 99-143.

Belgrand E. (1883) - Le Bassin parisien aux âges antéhistoriques. Paris, Imprimerie nationale, t. I, 288 p. et annexes, t. II, planches.

Bodu P., coordinateur (1995a) - le (loseau, Rucil-Malmaison (Hauts-de-Seine). Document final de synthèse de diagnostic, AFAN, SRA Ile-de-France. DDE des Hauts-de-Seine, 86 p.

Bodu P. (1995b) - Un gisement à Federmesser sur les bords de Seine : "le Closeau " à RucilMalmaison (Hauts-de-Seine). Bull. Soc. Préhicst. fr.. 92, n 4, 451-455.

Bohncke S. et Vandenberghe J. (1987) - Palaeohydrological development in the southern Netherlands during the last 1500() years in Starkel L., (Gregory K.J., Thornes J.-B. (edit.). Temperate Palaeohydrology. John Wiley, Chichester, 253-281.

Bohncke S., Vandenberghe J., Coope R. et Reiling R. (1987) - Geomorphology and palaeoccology of the Mark valley (southern Netherlands) : Palacoecology, palaeohydrology and climate during the Wejchselian Late Glacial. Boreas, 16, 69-84.

Bohncke S., Kasse C. et Vandenberghe J. (1995) - Climate induced environmental changes during the Vistulian Lateglacial at Zabinko, Poland. Quaestiones geographicae, special isue, 4, 43-64.

Bourdier F. (1969) - Etude comparée des dépôts quaternaires des bassins de la Seine et de la Somme. Bull. Info. Géologues Bassin de Paris. 21, 169-220.

Bravard J.-P., Peiry J.-L. et Gadiolet P. (1991) - La formation de la plaine alluviale holocène du Rhône à l'amont de Lyon. Physio-(jéo, 22-23, 167-172.

Bravard J.-P. (1992) - Les rythmes d'évolution morphologique des vallées françaises au Tardiglaciaire et à l'Holocène. Bull. Asssoc. (iéogr: Franç., Paris, 3, 207-226.

De Ploey J. (1963) - Palynological investigations of Upper Pleistocene and Holocene deposits in the Lower Kempenland (Belgium). Grana Palynologica, 4 (3), 428-438.

Emontspohl A.-F. et Vermeersch D. (1991) - Premier exemple d'une succession Bølling - Dryas II - Allernd en Picardie (Famechon, Somme). Quaternaire, 2. 17.25.

Fagnart J.-P. (1993) - Le Paléolithique supérieur récent et final du Nord de la France dans son cadre paléoclimatique. Thèse de doctorat, Université des Sciences et Techniques de Lille, 2 vol., $567 \mathrm{p}$.

Frenzel B., Vandenberghe J., Kasse K., Bohncke S. et Gläser B. edit. (1995) - European river activity and climatic change during the Lateglacial and early Holocene. Paläoklimaforschung/Palacoclimate Research, 14.

Gauthier A. (1995) - Résultats palynologiques de séquences holocènes du Bassin parisien : histoire de la végétation et action de l'Homme. Palynosciences, 3, 3-17.

Haesaerts P. (1984a) - Aspects de l'évolution du paysage et de l'environnement en Belgique au Quaternaire in Cahen D. et Haesaerts P. (dir) Peuples chasseurs de la Belgique dans leurs cadre naturel. Institut royal des Sciences naturelles de Belgique, Bruxelles, 28-39.

Haesaerts P. (1984h) - Les formations fluviatiles pléistocènes du bassin de la Haine (Belgique). Bulletin de l'Association Française pour l'Etude du Quaternaire, 21, 19-26.

Kalicky T. (1991) - The evolution of the Vistula River Valley between Cracow and Niepolomice in Late Vistulian and Holocene times in Starkel L. (edit.), Evolution of the Vistula River Valley during the last 15000) years. Polish Academy of Sciences, Wroclaw, 11-37.

Kerney M.P., Preece R.C. and Turner C. (1980) - Molluscan and plant biostratigraphy of some Late Devensian and Flandrian deposits in Kent. Philosophical transactions of the Royal Society of London, Serics B 291, 1-43.

Kerney M.P., Cameron R.A.D. und Jungbluth (1983) - Die Landschnecken Nord- und Mitteleuropas. Hamburg. Paul Parey, 384 p.

Kozarski S. (1983; - River channel adjustment to climatic change in west central Poland in Gregory (edit.). Background to Palaeohydrology. John Willey, Chichester, 355-374.

Kuzucuoglu C., Lespez L. and Pastre J.-F. (1992a) - Holocene colluvial deposits on the slopes of the Paris Basin in Bell M. and Boardman J. Eds. "Past and Present Soil Erosion ". Oxbow Monograph 22. $115-123$.

Kuzucuoglu C., Lespez L. and Pastre J.-F (1992b) - Can the magnitude and dynamics of colluvial deposits be related. through archatelogical data to land clearing and agriculture? 1st International Regional Conference on (ieomorphology (Natural Hazards) Proceedings. Ankara. 99-113. 
Krier V., Leroyer C. et Limondin N. (1991) - Approche pluridisciplinaire de l'environnement du site holocène de Verrières (vallée de la Scine, Aube). $116^{e}$ Congr. nat. des Soc. sav., Chambéry, Pré-protohistoire, 47-59.

Lautridou J.-P. (1985) - Le cycle pléniglaciaire en Europe du nord-ouest et plus particulièrement en Normandie. Thèse d'Etat en Géographic, Université de Caen, 908 p.

Leroyer C. (1995) - Fresnes-sur-Marne, le Chemin des Noues; Fresnes-sur-Marne l'Ile-du-Gord. Direction du Patrimoine, Sous-Direction de l'Archéologie, Bilan scientifique des centres nationaux 1993, 59-67.

Leroyer C. (1997) - Homme, climat, végétation au Tardi-et Postglaciaire dans le Bassin parisien : apports de l'étude palynologique des fonds de vallée. Thèse de doctorat de l'Université Paris I.

Leroyer C., Pastre J.-F., Fontugne M. et Limondin N. (1997) - Le Tardiglaciaire et le début de l'Holocène dans le bassin aval de la Marne (Seine-et-Marne, France) : chronostratigraphie et environnement des occupations humaines. In Le Tardiglaciaire en Europe du Nord-Ouest. Fagnart J.-P. et Thévenin A. Eds. Editions du CTHS, Paris, 151-164.

Lefèvre D., Heim J., Gilot J. et Mouthon J. (1993) - Evolution des environnements sédimentaires et biologiques à l'Holocène dans la plaine alluviale de la Meuse (Ardennes, France) : premiers résultats. Quaternaire, 4 (1), 17-30.

Limondin N. (1995 a) - Late-Glacial and Holocene malacofaunas from archaeological sites in the Somme Valley (North France). Journal of Archaeological Science, 22, 683-698.

Limondin N. (1995 b) - Analyse des malacofaunes. In : Bodu coordinateur : Le Closeau - RueilMalmaison (Hauts-de-Seine), 24-28.

Limondin N. (sous presse) - Les successions malacologiques du Tardiglaciaire et du début de l'Holocène dans la vallée de la Somme. $119^{\prime}$ congrès annuel des sociétés historiques et scientifiques, Amiens, 26-30 octobre 1994. Résumés des interventions et actes sous pressé.

Munaut A.V. et Paulissen E. (1973) - Evolution et paléoécologie de la vallée de la Petite Nèthe au cours du Post-Würm (Belgique). Ann. Soc. Géol. Belg., 96 (II), 301-346.

Pastre J.-F., Cecchini M., Dietrich A., Fontugne M., Gauthier A., Kuzucuoglu C., Leroyer C. et Limondin N. (1991) - L'évolution holocène des fonds de vallées au nord-est de la région parisienne (France) : premiers résultats. Physio-géo, $\mathrm{n}^{\circ} 22-23,109-115$.

Preece R.C. (1993) - Late Glacial and Post Glacial Molluscan successions from the site of the Channel Tunnel in SE England. Scripta Geologica, Special Issue 2, 387-395.

Roblin-Jouve A. (1994) - Le milieu physique In Taborin Y. (edit.). Environnements et habitats magdaléniens dans le centre du Bassin parisien. Documents d'Archéologie Française, Paris, n² 43, 12-35.

Starkel L., Gregory K.J., Thornes J.-B. edit. (1987) - Temperate Palaeohydrology. John Wiley, Chichester, $548 \mathrm{p}$.

Talon M., Bernard V., Billand G., Cottiaux R., Pastre J.-F., Pernaud J.-M. et Prost D. (1995) Le niveau organique Néolithique récent du site stratifié de la station d'épuration à La Croix-Saint-Ouen (Oisc). Premicrs résultats. Rev. archéo. Picardie, n spécial 9, 83-103.

Valladas H. (1981) - Datation par thermoluminescence de grès brulés de foyers de quatre gisements du Magdalénien final du Bassin Parisien. C.R. Acad. Sc. Paris, t. 292 série II, 355-358.

Valladas H. (1994) - Chronologic des sites du Magdalénien final du Bassin parisien In Taborin Y. (edit.). Environnements et habitats magdaléniens dans le centre du Bassin parisien. Documents d'Archéologie Française, Paris, 65-68.

Vandenberghe J. (1993) - Changing fluvial processes under changing periglacial conditions. Zeitschrift für Geomorphologie, Suppl. Bd 88, 17-18.

Vandenberghe J., Bohncke S., Lammers W., Zilverberg L. (1987) - Geomorphology and palaeoecology of the Mark valley (southern Netherlands) : Geomorphological valley development during the Weichseliean and Holocene. Boreas, 16, 55-67.

Vandenberghe J., Kasse C., Bohncke S. et Kozarski S. (1994) - Climate-related river activity at the Weichselian-Holocene transition : a comparative study of the Warta and Maas rivers. Terra nova, 6, 476485 .

Van Zeist W. and Van Der Spoel-Walvius M.R. (1980) - A palynological study of the Late-glacial and the Postglacial in the Paris basin. Palaeohistoria, XXII, 68-109.

Woillard G. (1975) - Recherches palynologiques sur le Pléistocène dans l'est de la Belgique et dans les Vosges lorraines. Acta Geog. Lov., 14, 118 p. 